\title{
Belgeo
}

Revue belge de géographie

$4 \mid 2019$

Les politiques de mobilité urbaine en Europe après la crise : adaptations et innovations

\section{Patrick Rérat, Gianluigi Giacomel, Antonio Martin, Au travail en vélo. La pratique utilitaire de la bicyclette en Suisse}

Collection Espaces, mobilités et sociétés, Editions Alphil-Presses

Universitaires Suisses, 2019

\section{Mathieu Strale}

\section{OpenEdition}

Journals

Édition électronique

URL : http://journals.openedition.org/belgeo/35279

DOI : 10.4000/belgeo.35279

ISSN : 2294-9135

Éditeur :

National Committee of Geography of Belgium, Société Royale Belge de Géographie

Référence électronique

Mathieu Strale, «Patrick Rérat, Gianluigi Giacomel, Antonio Martin, Au travail en vélo. La pratique utilitaire de la bicyclette en Suisse », Belgeo [En ligne], 4 | 2019, mis en ligne le 05 novembre 2019, consulté le 24 septembre 2020. URL : http://journals.openedition.org/belgeo/35279 ; DOI : https:// doi.org/10.4000/belgeo.35279

Ce document a été généré automatiquement le 24 septembre 2020.

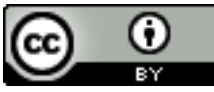

Belgeo est mis à disposition selon les termes de la licence Creative Commons Attribution 4.0 International. 


\section{Patrick Rérat, Gianluigi Giacomel, Antonio Martin, Au travail en vélo. La pratique utilitaire de la bicyclette en \\ Suisse}

Collection Espaces, mobilités et sociétés, Editions Alphil-Presses

Universitaires Suisses, 2019

Mathieu Strale

\section{RÉFÉRENCE}

Patrick Rérat, Gianluigi Giacomel, Antonio Martin, Au travail en vélo. La pratique utilitaire de la bicyclette en Suisse, Collection Espaces, mobilités et sociétés, Editions Alphil-Presses Universitaires Suisses, 2019

1 Cet ouvrage, septième volume de la collection Espaces, mobilités et sociétés des Presses universitaires suisses, traite de la pratique du vélo en Suisse, en s'appuyant sur une enquête menée auprès d'un peu moins de 14000 participants à l'action de promotion des déplacements cyclables sur le chemin du travail Bike to work. L'objectif est d'identifier et comprendre les pratiques différenciées du vélo dans la population, les facteurs d'attraction ou au contraire les obstacles à cette pratique et l'impact des politiques publiques.

2 L'analyse commence par une brève introduction historique sur l'usage du vélo, en particulier en Suisse. Celle-ci témoigne d'une pratique ayant connu un essor récent après un déclin tout au long de la seconde moitié du vingtième siècle et d'un usage qui reste relativement modeste en Suisse en comparaison des pays voisins, en particulier du nord. Sont aussi soulignés les avantages environnementaux, sociaux et de santé publique de la pratique cycliste. 
3 Les auteurs entrent ensuite dans le vif du sujet en présentant leur grille d'analyse des facteurs influençant la pratique du vélo, à savoir le potentiel de mobilité des individus et le potentiel d'accueil du territoire. Le potentiel de mobilité des individus regroupe la question de l'accès au vélo, que ce soit par le biais de la propriété ou du partage, des compétences, c'est-à-dire la capacité à se déplacer aisément en vélo et l'appropriation, soit la perception et la sélection des modes de transports dont le vélo. Au niveau du potentiel d'accueil des territoires, les enjeux sont le contexte spatial, qui regroupe notamment le relief et le climat mais aussi l'aménagement du territoire et les formes urbaines, les infrastructures et aménagements prévus pour les vélos, au niveau des axes de circulation mais aussi du stationnement et de l'entretien et enfin les normes et règles qui s'appliquent à l'usage du vélo. Cette grille d'analyse sert de trame à la suite.

4 Le chapitre 3 traite de la pratique du vélo en Suisse sur base des statistiques nationales et met en évidence d'importants clivages entre Suisse alémanique, romande et italienne, avec une pratique plus forte dans la première, ainsi qu'entre grandes villes, où l'usage du vélo est plus important, et espaces périurbains, de petites villes et ruraux. Les auteurs signalent aussi la grande variabilité, y compris entre villes de taille similaire et au sein de cantons relevant du même groupe linguistique. Du point de vue sociologique, le vélo apparaît comme une pratique de jeunes adultes urbains se mouvant pour se rendre au travail ou sur un lieu de formation.

5 Ensuite est présentée l'enquête Bike to work, qui est un questionnaire soumis chaque année depuis 2005 à un panel de travailleurs répartis sur l'ensemble du territoire suisse. La suite de l'ouvrage s'appuie sur les résultats du millésime 2016, qui a permis de récolter un peu moins de 14000 réponses validées sur 54000 envois. L'analyse étant centrée sur les travailleurs utilisant le vélo, les classes d'âge jeunes et âgées, les femmes et les personnes peu diplômées sont donc sous-représentées dans l'échantillon. Au-delà d'une enquête, l'initiative Bike to work vise aussi à promouvoir l'usage du vélo en menant des actions de publicité et de sensibilisation.

6 De l'analyse des déplacements ressort une pratique du vélo qui se concentre sur les déplacements domicile-travail de moins de 30 minutes. Les auteurs s'attachent dans ce cadre à différencier les cyclistes, allant de l'usager occasionnel au cycliste systématique. Les résultats confirment l'image d'une pratique urbaine, de ménages sans enfants. S'agissant de l'accès au vélo, les auteurs soulignent l'importance de l'émergence des vélos à assistance électrique qui permettrait d'élargir socialement la pratique à des personnes plus âgées, moins urbaines et moins masculines.

7 En ce qui concerne les compétences les auteurs soulignent l'importance des aménagements améliorant la sécurité réelle et perçue des cyclistes pour élargir la pratique du vélo à de nouvelles catégories de populations. Au travers du suivi de panels depuis le début de l'action Bike to work apparaît aussi l'impact de l'apprentissage et de l'expérience, qui rend la perception des déplacements cyclables plus positive du point de vue de la sécurité, de la pénibilité physique ou de l'aisance à circuler.

8 Les motivations de l'usage du vélo sont ensuite analysées. Une classification en quatre catégories est établie: les cyclistes actifs, motivés par leur bien-être physique et psychique, les cyclistes civiques, sensibles aux enjeux environnementaux et sociétaux, les cyclistes individualistes motivés par leur bien-être mais aussi leur plus grande indépendance dans les déplacements, et enfin les cyclistes enthousiastes, qui valorisent tant les facteurs individuels que collectifs. 
9 De l'enquête ressortent aussi des freins à la pratique du vélo. Ceux-ci sont d'ordre climatique, en particulier en lien avec les hivers pouvant être rudes en montagne, ou encore logistique, c'est-à-dire la nécessité de transporter des charges, de combiner des déplacements multiples ou de transporter des enfants, sécuritaires et de confort.

Du point de vue territorial, l'enquête semble confirmer les clivages déjà cités, à savoir une pratique du vélo urbaine et concentrée dans les cantons germanophones. Ces différences tiendraient à la qualité des aménagements mais aussi à la perception sociétale de l'usage du vélo.

11 Logiquement, l'évaluation des politiques publiques en matière de promotion du vélo suit un clivage similaire, avec une perception plus positive dans les villes et cantons germanophones, alors qu'elle est mauvaise en Suisse romande et italienne. Le lien est ainsi établi entre sentiment de sécurité et satisfaction quant aux politiques menées en la matière.

12 L'analyse se conclut par une série de recommandations pour favoriser l'usage du vélo, du point de vue de la promotion de la pratique et des aménagements nécessaires pour sécuriser et faciliter ces déplacements.

13 De cet ouvrage ressort donc la diversité des pratiques cyclistes, en particulier dans le cadre suisse des déplacements domicile-travail. L'approche méthodologique est solide et s'appuie sur une enquête d'assez grande ampleur. On peut néanmoins regretter la faible prise en compte des enjeux sociaux. Ainsi, très peu d'informations sont données quant au lien entre conditions sociales et usage du vélo : impact du niveau de revenu et des conditions de vie, de la profession, de la composition du ménage, de l'accessibilité aux services de base, enjeux des déplacements contraints... Il serait intéressant d'intégrer ces dimensions, dont l'impact est reconnu sur la répartition modale des déplacements dans les futurs opus de l'enquête suisse Bike to work. 